\title{
A HUGE LESION IN THE MAXILLARY POSTERIOR REGION DIAGNOSED HIGH GRADE MUCOEPIDERMOID CARCINOMA OF MINOR SALIVARY GLANDS: A CASE REPORT
}

\author{
Guldane Magat ${ }^{1}$, Sevgi Ozcan², Fatma Yuce ${ }^{1}$, Mine Cetin ${ }^{1}$, Mehmet Esad \\ Guven $^{3}$, Fakih Cihat Eravcı ${ }^{4}$, Kaan Orhan ${ }^{5}$
}

\footnotetext{
${ }^{1}$ Necmettin Erbakan University, Faculty of Dentistry, Department of Oral and Maxillofacial Radiology, Konya, Turkey

${ }^{2} \mathrm{Hacı}$ Bektas Veli University, Faculty of Dentistry, Department of Oral and Maxillofacial Radiology, Nevsehir, Turkey

${ }^{3}$ Necmettin Erbakan University, Faculty of Dentistry, Department of Prosthodontics, Konya, Turkey

${ }^{4}$ Necmettin Erbakan University, Meram Faculty of Medicine, Department of Otolaryngology, Konya, Turkey

${ }^{5}$ Ankara University, Faculty of Dentistry, Department of Oral and Maxillofacial Radiology, Ankara, Turkey
}

Address for Correspondence: Guldane Magat, E-mail: gul_dent@hotmail.com Received: 01.11.2021; Accepted: 11.01.2022; Available Online Date: 27.01 .2022

CCopyright 2021 by Dokuz Eylül University, Institute of Health Sciences - Available online at https://dergipark.org.tr/en/pub/jbachs

Cite this article as: Magat G, Ozcan S, Yuce F, Cetin M, Guven ME, Eravcı FC, Orhan K. A Huge Lesion in the Maxillary Posterior Region Diagnosed High Grade Mucoepidermoid Carcinoma of Minor Salivary Glands: A Case Report. J Basic Clin Health Sci 2022; 6: 306-313.

\begin{abstract}
Mucoepidermoid carcinoma (MEC) is the most common malignant tumor of the salivary glands. These tumors occur commonly in the major salivary glands, especially the parotid, and they are the most frequently located in the palate intraorally. The similarity between MEC and other odontogenic cysts in the mouth is a critical situation that needs attention in clinical examination. Early diagnosis is very important in the diagnosis of asymptomatic growth, reaching very large sizes easily, its recurrence potential, and malignant character. The treatment and prosthetic rehabilitation of MEC are specified according to histopathological grade, location, and invasion pattern of the tumors. The purpose of this case is to present a 19-years-old female with high-grade MEC of minor salivary glands at the palate.
\end{abstract}

Key Words: mucoepidermoid, carcinoma, cone-beam computed tomography, diagnostic imaging.

\section{INTRODUCTION}

Salivary gland tumors account for $3 \%$ of head and neck tumors. Mucoepidermoid carcinoma (MEC) is the most common (12-29\%) malignant salivary gland tumor. Fifty percent of the cases occur in the major salivary glands; about $80 \%$ parotid, $8-23 \%$ submandibular, $2-4 \%$ sublingual (1). In addition, it is mainly located on the palate in case of the occurrence of MEC in minor salivary glands. MEC occurs more frequently between 3-6 decades although it can appear at all ages. The prevalence of MEC for females is more compared with males in 3:2 ratios. MEC may also exhibit aggressive behavior besides clinically a slow growth pattern. On palpation, MEC is soft or rubbery, with or without a handle, and it is also usually painless (2). MEC is classified as low-grade, medium-grade, and high-grade according to cytological features, invasion pattern, and cellular type (3). Histopathological grading is to determine the lesion size, cystic component, nuclear atypia, and necrosis content. The treatment regimens depend on histopathological grade, clinical features, and localization. Standard surgical resection and combined adjuvant therapy are used to reduce the risk of recurrence in salivary gland cancers (1). In this article, a case of high-grade MEC detected in the 


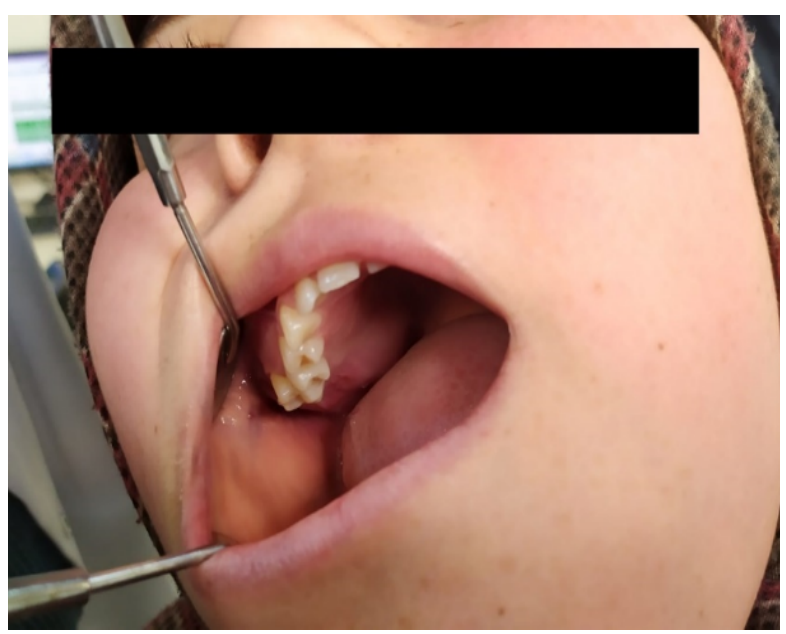

Figure 1. The intraoral view shows a non-tender swelling with a smooth surface in the palate area.

palate region of a 19-year-old asymptomatic patient is presented.

\section{Case Report}

A 19-year-old female patient of Turkish origin reported to the Department of Oral and Maxillofacial Radiology, Necmettin Erbakan University, Konya, Turkey, with the chief complaint of a swelling in the right maxillary region that has been continuing for 5 years. She stated that the lesion had increased gradually with no pain. The oral hygiene status of the subject was satisfactory. The patient did not have a history of cigarette smoking, alcoholism, or "betel quid" use. No significant medical history related patient and her family were noted. There were no previous radiographic images of this patient.

On extraoral examination, a single swelling in the right maxillary region extending horizontally from the ala of the nose to the zygomatic region and vertically from the level of corner of the mouth to the infraorbital margin was noticed. The skin over the swelling was normal. The swelling was non-tender. The submandibular and cervical lymph nodes were normal clinically.

Intraoral examination revealed that there was nontender and painless swelling in the upper right buccal vestibule covered with normal mucosa. Electrical pulp test results were positive for maxillary posterior teeth. Clinically the second upper right molar was missing, and the covering mucosa was intact, too (Figure 1). Two separate lesions were detected in panoramic radiography. While there was a distinctly demarcated unilocular radiolucent lesion between the canine tooth and the mesial root of the first molar tooth, a multilocular radiopaque-radiolucent lesion with unclear borders starting from the distal root of the first molar tooth, including the retromaxillary region and extending to the orbit was detected. The borders of the maxillary sinus floor, orbital floor, and lateral wall of the nasal cavity could not be clearly observed. The lesion contained the crown of the impacted maxillary 3rd molar tooth (Figure 2).

Cone-beam computerized tomography ( $\mathrm{CBCT}$ ) scans were obtained and stated a huge mass of $49 \times 33 \times 35$ $\mathrm{mm}$ invading the right maxillary sinus. There was significant bone destruction with cortical bone expansion. While perforation and expansion were detected on the buccal cortical plate, the lesion uplifted of the roof of the maxillary sinus without evidence of invasion to the floor of the orbit. Sclerosis formation resembling root residue was detected between the 2nd premolar and 1st molar teeth. Also, it was detected that a lesion involved the crown of the impacted third molar (Figure 3). For the evaluation of soft tissues, computerized tomography (CT) was taken. CT revealed the dehiscences and spread of the lesion to the surrounding soft tissues. It was also detected that the lesion was spread into the pterygopalatine fossa, nasal cavity, and the foramen rotundum region (Figure 4).

Evaluating the clinical and radiographic findings, the provisional diagnosis considered was ameloblastoma. A biopsy from the lesion was done by oral and maxillofacial surgery. The first histopathology result obtained from the sample taken in the Oral and Maxillofacial Surgery Department was ameloblastoma. The patient was marsupialized according to the ameloblastoma result. However, no reduction in lesion size was detected. The patient was then referred to the Otolaryngology Department. The complete surgical resection of the mass was performed with Class $2 \mathrm{~b}$ maxillectomy (leaving the orbital roof) according to the Brown classification by the Otolaryngology Department. The definitive histopathological evaluation of the mass revealed a high-grade mucoepidermoid carcinoma. The patient has been on a regular follow-up for one year. In the postoperative Magnetic Resonance Imaging (MRI), no malignancy recurrence was observed in the tumor resection bed, while edema was detected in the nasal cavity (Figure 5). Approximately 3 months after 
surgery, the patient was consulted to the Prosthodontics Department for a permanent maxillary obturator. Due to the size of the defect (Figure $\mathbf{6} \mathbf{a}, \mathbf{b})$ and both aesthetic and functional needs, a precision attachment maxillary obturator was planned for the patient. 2-unit fixed partial dentures restorations were applied in the anterior and posterior segments for the placement of retaining attachments. A precision attachment maxillary obturator prosthesis was produced that was placed on the holder parts on these restorations (Figure $6 \mathbf{c , d}$ ). In addition, the patient was given oral hygiene information again due to insufficient tooth brushing habits. Six months after the prosthesis was applied, the patient was called for control. Retention and phonation tests were applied.
No oral-nasal liquid leakage was observed. Also, a significant improvement was observed in the pronunciation of palata-dorsal sounds (/t/, /d/, /n/, /l/, $/ \mathrm{k} /, / \mathrm{g} /, / \mathrm{n} /, \mathrm{s} /, \mathrm{z} /)$. And the patient began to maxillary obturator wear due to a postoperative maxillary defect. No sign of recurrence was found in the MRI taken at the patient's follow-up 6 months later.

\section{DISCUSSION}

MEC has been stated as the most common malignancy of the salivary glands in the literature and usually occurs in the major salivary glands and is the most common location is the parotid gland (80\%). When it is in the minor salivary glands, the palate is the most frequent site. Except for the palate, it may

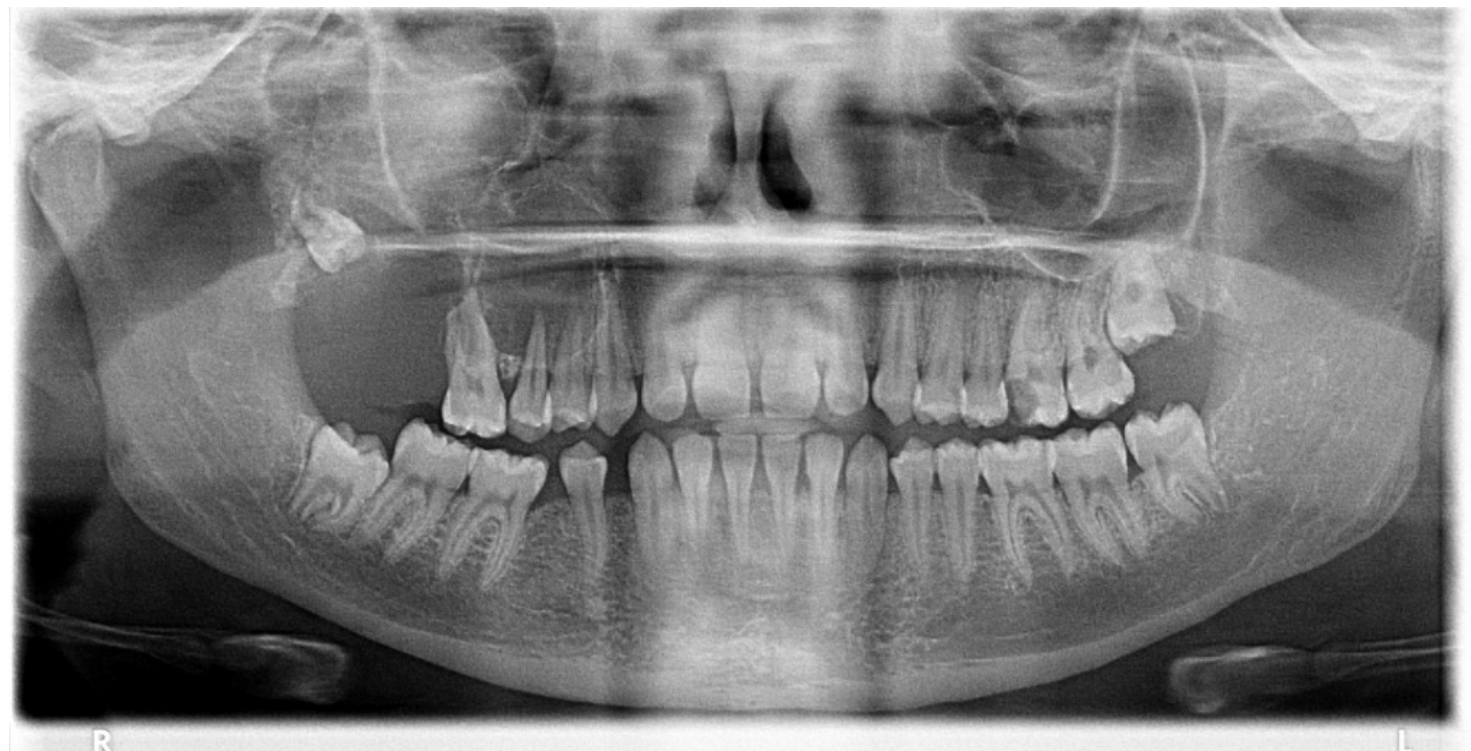

Figure 2. Panoramic radiography shows two separate lesions in the right maxillary region. While the first lesion was in the form of a clearly circumscribed unilocular radiolucent lesion between the canine tooth and the mesial root of the first molar tooth, the second lesion was multilocular radiopaque-radiolucent with unclear borders, starting from the distal root of the first molar and including the retromaxillary region, including the crown of the maxillary third molar tooth.

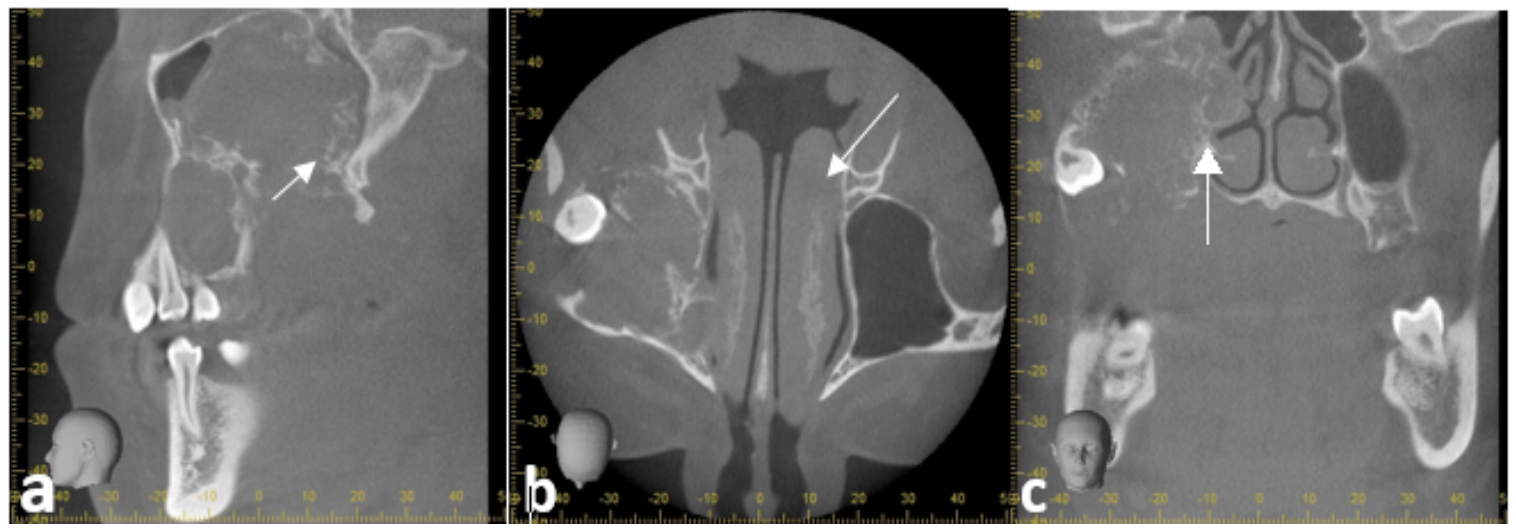

Figure 3. $\mathrm{CBCT}$ images showing an expansive very huge lesion invading the right maxillary sinus and nasal cavity on sagittal (a), axial (b) and coronal (c) slice. 
appear in the buccal mucosa, alveolar mucosa, retromolar region, lips, tongue, and floor of the mouth. Apart from the salivary glands, it may occur in the esophagus, thyroid, breast, lung, and pancreas (4). MEC occurs between the ages of 8-92 but it may develop at any age. A recent study has reported that the average age was 48.8 and peaks in the $5^{\text {th }}$ decades of life (5). However, it is characteristically the most common childhood malignancy (6). In the pediatric and adolescent population, most malignant salivary gland tumors are diagnosed at an average age of 13.5 years, while benign neoplasms occur at a slightly older age (15 years) (4). Sultan et al. (7) emphasized that a solitary salivary gland neoplasm is more likely to be malignant in a child than in an adult. In addition, there was no difference between males

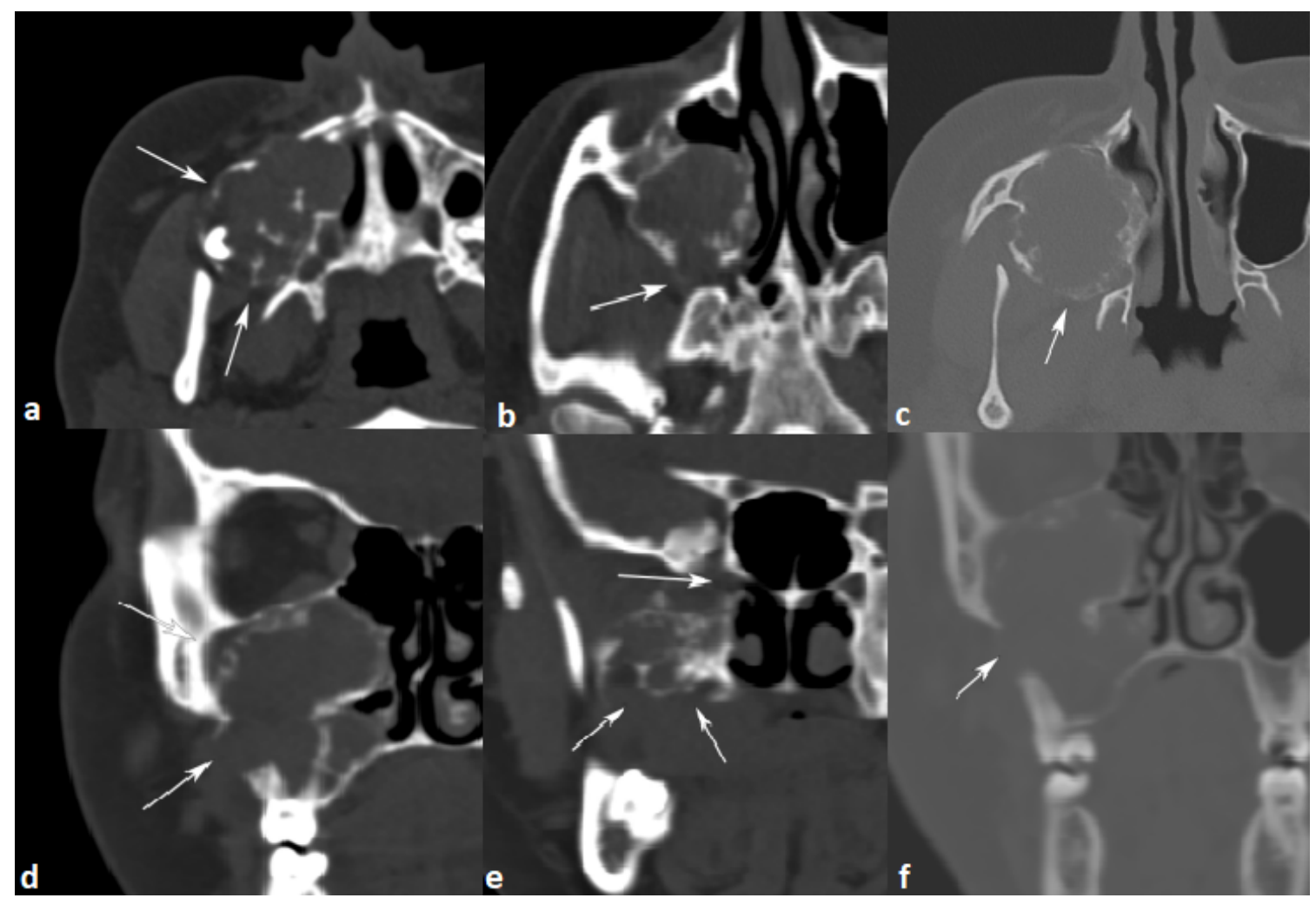

Figure 4. (a) Axial CT (soft tissue window) showing an expansive lesion in the right maxilla, Note that the dehiscences and spread of the lesion to the surrounding soft tissues (arrows), (b) Axial CT also shows the spread of the lesion into the pterygopalatine fossa (arrows), (c) Axial CT (bone tissue window) showing expansion and sclerosis nature of the lesion, (d) Coronal (soft tissue window) showing the infiltration of the lesion into the maxillary sinus (arrows), e) Note the tumoral lesion infiltrating for. rotundum (arrows), (F (Coronal CT (bone tissue window) showing the perforation of the maxilla and root resorption.

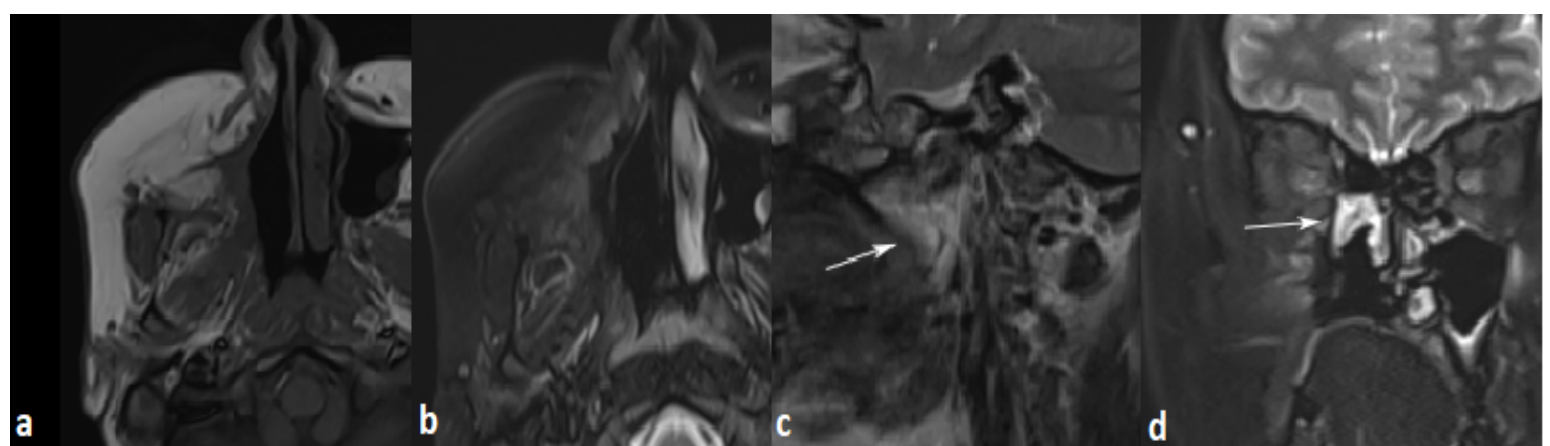

Figure 5. Post-Op MRI s showing, (a) Axial T1-w image showing the surgical defect, (b) Axial T2-W postop defect without remnant tumoral lesion, (c,d) Sagittal and Coronal t'-W images showing nasal cavity edema sourced from mucosa (arrows) without any malignancy. No areas of contrast enhancement were detected. 
and females in terms of high-grade MEC. However, it appears more in older ages (51.2) as compared to low grades (35.7) (2). In our case, the patient's age, gender, and occurring site suitable typically MEC behaviors.

According to the proportion of cell types in MEC contain, it is classified as low-, intermediate-, and high-grade pathologically. Low-grade MEC consists of well-differentiated, by a majority of mucussecreting cells and glandular structures. On the other hand, high-grade MEC consists of poorly differentiated squamous cells with poorly circumscribed solid character and secreting less mucus. So high-grade tumor behavior is generally infiltrative. Perineural invasion and necrosis also could be seen (4). Low-grade and moderate MEC were the most encountered in studies reported (30.4$66.7 \%, 38.4-75 \%)$. The incidence of high-grade MEC varies between $3.2-63.7 \%$. After evaluation MEC is thought of as benign if it is during the early stages; whereas in advanced level it has a very aggressive prognosis (8). Spiro et al. (9) reported that survival was importantly affected by the histopathologic grade and clinical stage in minor salivary gland malignancies. Therefore, the 5 -year survival rate of high-grade MEC is $26 \%$, low-grade MEC may go up to $90-100 \%$. In the post-surgical MRI examination, perineural invasion and necrosis were not detected in our case.

Low-grade malignancies are characterized by slowgrowing swelling without pain. Otherwise in highgrade lesions, rapid growth, invasion to adjacent tissue, metastasis, and ulceration may be seen. Apart from swelling, mass and pain are other symptoms commonly findings (4). Li et all. (10) have reported that low-grade lesions generally (\%76.2) display symptoms and in addition, the swelling is $\% 91.1$ of all symptoms. Generally, high-grade lesions show many different symptoms. In this case, besides intraoral swelling, no clinical symptoms or findings were presented. Therefore, only one symptom existing is very rare just like our case. According to Armed Forces Institute of Pathology (AFIP) cases, tumor size range between less than $1 \mathrm{~cm}$ to more than 12 $\mathrm{cm}$ for major salivary glands. Besides that, minor salivary glands diameter as large as $5 \mathrm{~cm}$. Russell et al. (11) stated that tumors larger than T1 stage have a higher risk of metastasis, but MECs smaller than 2.5 $\mathrm{cm}$ are rarely fatal regardless of grade. In our case, the size of the tumor was approximately $5 \mathrm{~cm}$.

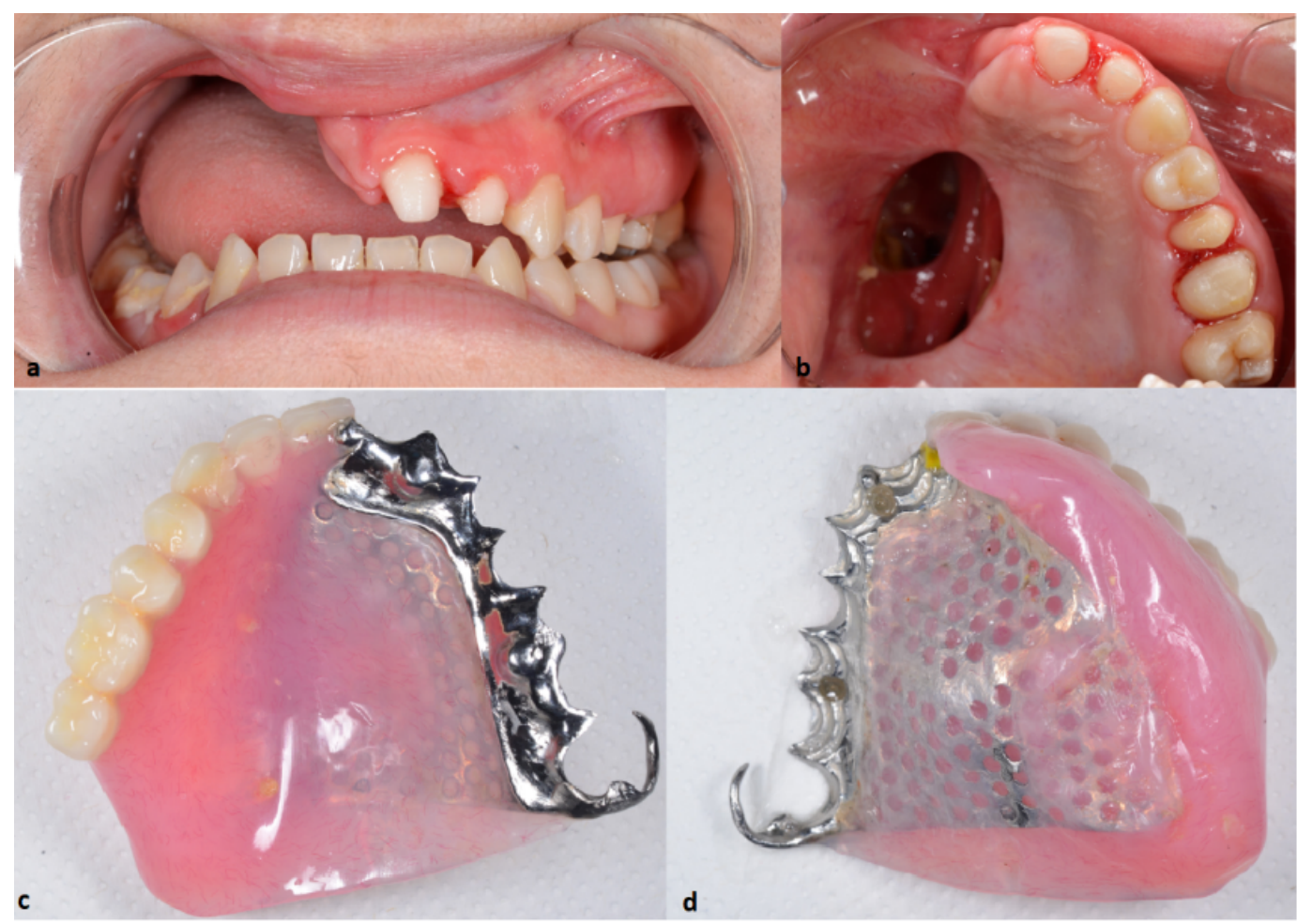

Figure 6. Postoperative intraoral and prosthetic restoration images (a) Sagittal intraoral view approximately 3 months after surgery, (b) Intraoral view from the palatal aspect, $(c, d)$ inner and outer part of the obturator. 
Although the lesion size was $5 \mathrm{~cm}$ and the histopathologic result was high grade, the only clinical finding was intraoral swelling in the present case. Therefore, it should be kept in mind that clinical diagnosis can be misleading in the palatal localization of MEC.

Swelling is the most common symptom regardless of the tumor grades (4). The patient thought that swelling occurred after tooth extraction 5 years ago. Also, no pain, ulceration, or lymphadenopathy was observed. Pain is not encountered in MEC cases, but unfortunately, there are generally high-grade cases with lymphadenopathy, ulceration, and bone involvement. Early diagnosis of malignant lesions to be advantaged for the treatment and the effectiveness of treatment. But unfortunately, the absence of symptoms may cause a delay in diagnosis and a decrease in the effectiveness of treatment. The clinical features of our case showed that biopsy is essential for diagnosis, and clinical diagnosis may be misleading. Therefore, oral lesions may mimic each other and present very different clinical manifestations (12). In addition, just only examining radiologic images does not lead us to correct diagnosis, but it is essential for a total examination.

Radiologically, the MEC can show many different images. The image may not be detected on standard panoramic radiography or the radiopaque-radiolucent image may be seen clearly. Unlike panoramic radiograph, $\mathrm{CBCT}$ provided valuable information to diagnose the lesion as the tooth roots were resorbed and showed the perforation or resorption where and how. CT and MR images may change depending on the grade of the tumor. CT is usually used to extent of masses, evaluate morphology, and assess lymphadenopathy and bone involvement. MR is used to assess evaluate for perineural invasion and the extent of a tumor. MR images of low-grade MEC are markedly hyperintense on T1-T2-weighted images. High-grade MEC lesions on T2-weighted images may show hypointense or isointense (13).

Odontogenic keratocyst, ameloblastoma, dentigerous cyst, ameloblastic fibroma, glandular odontogenic cyst, primary intraosseos carcinoma, and other malignant salivary glands tumors should be considered in the radiological differential diagnosis of MEC $(14,15)$. Histological diagnosis of high-grade MEC developing in an odontogenic cyst requires a differential diagnosis from other intraosseous lesions, which may be similar to a cyst in clinical examinations. Morphological features of the epithelial lining provide the grounds for eliminating odontogenic cysts. Epithelium of true cysts of maxillary bones is not atypical, therefore dentigerous, radicular, or orthokeratotic cysts might be easily excluded. A glandular odontogenic cyst is the most important entity in the differential diagnosis of central MEC because of their morphological similarity. Also, some low-grade central MECs would have originated from glandular odontogenic cysts (16). Kaplan et al. (17) defined major and minor criteria for the diagnosis of the glandular odontogenic cyst. They suggested that the major criteria are mandatory, whereas the minor criteria do not need to be present for diagnosis, but may support it. Our case was considered to be MEC because the presence of nests and islands of epidermoid, mucous, and intermediate cells showing cystic spaces in a fibrous stroma, and mucin demonstrated by mucicarmine stain supports the diagnosis of MEC in this case. Histologically, this case did not meet the major criteria of the glandular odontogenic cyst.

Standard treatment of salivary gland cancers is surgical resection, and combined adjuvant therapy. The localization, clinical features, and histopathology of MEC are effects to determine the treatment method. Also the treatment protocol should be determined specifically for each case. Radical surgery is adequate for low-grade MEC and adjuvant therapy is considered for high-grade lesions. Generally, wide surgical excision is sufficient. However, adjuvant therapy is indicated in the presence of positive margins, metastasis to the lymph nodes, or residual disease (18). The latest review of Li et al.(10) reported that \%33.3 of high-grade MEC were performed only in surgery and \%60 of them were operated on surgery + radiotherapy. The local recurrence rate may be reduced by aggressive surgical excision. In low-grade tumors, no recurrence is observed after aggressive surgical excision. The high grade of the tumor, its size covering a large area, showing bone involvement, cervical lymph node metastasis, positive margins, or perineural invasion increase the possibility of recurrence and require postoperative radiotherapy. However, the postoperative effect of chemotherapy and/or radiotherapy in patients is due to the long-term side effects of radiotherapy. Chemotherapy should be considered for patients with local aggressive or metastases who are not suitable for surgery and radiotherapy (19). In our case, radiotherapy was not applied to the patient who underwent aggressive 
surgical resection. The patient has been followed up for recurrence for 6 months. No malignancy, perineural invasion or recurrence was detected in the MRI examination 6 months later.

Salivary gland malignancies have a wide range of prognoses depending on their histopathology. In general, the prognosis of salivary malignancies is better in children and adolescents than in adults due to the lower frequency of cervical metastases, the absence of local soft tissue invasion, and different histology. Regardless of the malignant subtype in the literature, adverse prognostic factors for survival include ethnicity, advanced age, male gender, highstage disease, high-grade histology, cervical metastasis, presence of pain, facial nerve involvement, perineural invasion, local soft tissue invasion, positive or near margins, distant metastases, and comorbidities have been shown (4). Our case was a young adult woman and had no cervical metastasis, facial nerve involvement, or perineural invasion. In some studies, smoking and alcohol use were stated to be poor prognostic factors. Our case also did not have a habit of smoking and alcohol use. Distant metastasis most commonly involves lungs $(40 \%$ to $91 \%)$, then bone $(13 \%$ to $40 \%$ ), liver (4\% to $19 \%)$, soft tissue $(9 \%)$, distant nodal nodes $(8 \%)$, and brain $(4 \%$ to $7 \%)$. It is one of the main factors in determining the survival of patients. In patients with minor salivary gland tumors, if distant metastases are present, the average survival rate is 2.3 years for the minor salivary glands and 2.6 years for the major salivary glands. Distant metastasis was not detected in our case, either. In addition, the metastasis of the tumor is related to its location. When the tumor is located in the palate, these metastases are usually local (4).

\section{CONCLUSION}

Although MEC is a malign salivary gland tumor, it may mimic other benign lesions and display a benign clinical appearance. Therefore, although osteolytic lesions have no clinical or radiological findings, they should be considered in terms of MEC. And we suggest that long-term follow-up due to aggressive behaviors and recurrence potential, especially at lesion with the high-grade level. Also, clinicians have to look suspiciously at a lesion in advanced clinical appearance in the palatal region and should check all image protocol and examine it carefully.
Author Contribution: All authors made substantial contributions to conception and design, and/or acquisition of data, and/or analysis and interpretation of data, and participated in drafting the article or revising it critically for important intellectual content, and all authors gave final approval of the version to be submitted and any revised version

Conflict of Interest: Authors have no conflict of interests, and the work was not supported or funded by any drug company.

Peer review: Externally peer reviewed.

\section{REFERENCES}

1. López-Terrada D, Bloom M, Cagle PT, Ostrowski ML. Oncocytic mucoepidermoid carcinoma of the trachea. Archives of Pathology and Laboratory Medicine. 1999;123(7):635-7.

2. Nance MA, Seethala RR, Wang Y, Chiosea SI, Myers EN, Johnson JT, et al. Treatment and survival outcomes based on histologic grading in patients with head and neck mucoepidermoid carcinoma. Cancer. 2008;113(8):2082-9.

3. Ellis MA, Graboyes EM, Day TA, Neskey DM. Prognostic factors and occult nodal disease in mucoepidermoid carcinoma of the oral cavity and oropharynx: an analysis of the National Cancer Database. Oral oncology. 2017;72:174-8.

4. Peraza A, Gómez R, Beltran J, Amarista FJ. Mucoepidermoid carcinoma. An update and review of the literature. Journal of stomatology, oral and maxillofacial surgery. 2020.

5. Schwarz S, Stiegler C, Müller M, Ettl T, Brockhoff G, Zenk J, et al. Salivary gland mucoepidermoid carcinoma is a clinically, morphologically and genetically heterogeneous entity: a clinicopathological study of 40 cases with emphasis on grading, histological variants and presence of the $t(11 ; 19)$ translocation. Histopathology. 2011;58(4):557-70.

6. Brandwein MS, Ivanov K, Wallace DI, Hille JJ, Wang B, Fahmy A, et al. Mucoepidermoid carcinoma: a clinicopathologic study of 80 patients with special reference to histological grading. The American journal of surgical pathology. 2001;25(7):835-45.

7. Sultan I, Rodriguez-Galindo C, Al-Sharabati S, Guzzo M, Casanova M, Ferrari A. Salivary gland carcinomas in children and adolescents: a population-based study, with comparison to adult cases. Head Neck. 2011;33(10):1476-81.

8. Poletto AG, Mello FW, Melo G, Rivero ERC.

Prevalence of mucoepidermoid carcinoma among intraoral minor salivary gland tumors: a 
systematic review and meta-analysis. Journal of Oral Pathology \& Medicine. 2020.

9. Spiro RH, Huvos AG, Berk R, Strong EW. Mucoepidermoid carcinoma of salivary gland origin: a clinicopathologic study of 367 cases. The American Journal of Surgery. 1978;136(4):461-8.

10. Li X, Wang F, Wang Y, Sun S, Yang H. An unusual case of intraosseous mucoepidermoid carcinoma of the mandible: A case report and literature review. Medicine. 2018;97(51).

11. Russell JL, Chen NW, Ortiz SJ, Schrank TP, Kuo YF, Resto VA. Racial and Ethnic Disparities in Salivary Gland Cancer Survival. JAMA otolaryngology-- head \& neck surgery. 2014;140(6):504-12.

12. da Silva L, Serpa M, da Silva L, Sobral A. Central mucoepidermoid carcinoma radiographically mimicking an odontogenic tumor: A case report and literature review. Journal of Oral and Maxillofacial Pathology. 2016;20(3):518-22.

13. Kato $H$, Kanematsu $M$, Makita $H$, Kato $K$, Hatakeyama D, Shibata T, et al. CT and MR imaging findings of palatal tumors. European journal of radiology. 2014;83(3):e137-e46.

14. Darling MR, Wehrli BM, Ciavarro C, Daley TD. Pericoronal radiolucency in the posterior mandible. Oral surgery, oral medicine, oral pathology, oral radiology, and endodontics. 2008;105(2):139-43.

15. White SC, Pharoah MJ. Oral radiology-E-Book: Principles and interpretation: Elsevier Health Sciences; 2014.

16. Brookstone MS, Huvos AG. Central salivary gland tumors of the maxilla and mandible: a clinicopathologic study of 11 cases with an analysis of the literature. Journal of Oral and Maxillofacial Surgery. 1992;50(3):229-36.

17. Kaplan I, Anavi Y, Hirshberg A. Glandular odontogenic cyst: a challenge in diagnosis and treatment. Oral diseases. 2008;14(7):575-81.

18. Liu S, Ow A, Ruan M, Yang W, Zhang C, Wang $\mathrm{L}$, et al. Prognostic factors in primary salivary gland mucoepidermoid carcinoma: an analysis of 376 cases in an Eastern Chinese population. International journal of oral and maxillofacial surgery. 2014;43(6):667-73.

19. Ali SA, Memon AS, Shaik NA, Soomro AG. Mucoepidermoid carcinoma of parotid presenting as unilocular cyst. J Ayub Med Coll Abbottabad. 2008;20(2):141-43. 